\title{
Avaliação de usabilidade do site SciELO e UFPR do Periódico Boletim de Ciências Geodésicas (BCG)
}

\author{
Evaluation of the usability of the Bulletin of Geodesic Sciences (BCG) \\ Vinicius Emmel Martins \\ Mestrando em Ciências Geodésicas \\ Universidade Federal do Paraná \\ vinicius.emme.m.92@gmail.com \\ Marcio Augusto Reolon Schmidt \\ Doutor em Ciências Geodésicas \\ Universidade Federal do Paraná \\ marcioarschmidt@gmail.com
}

\begin{abstract}
Resumo
O presente estudo apresenta uma abordagem estruturada em resultados obtidos com a aplicações de avaliações de usabilidade dos Web sites pertencentes ao periódico Boletim de Ciências Geodésicas (BCG), o BCG possui dois sites para comunicação com os autores e leitores, um faz parte da Scielo e outro pertence a Universidade Federal do Paraná (UFPR), que mantém sites para todo seu conjunto revistas, incluindo o BCG. A premissa estabelecida para realização do estudo foi que resultados distintos são obtidos com a aplicação de avaliações de usabilidade em ambos os sites. O objetivo do estudo foi avaliar a usabilidade do site da biblioteca digital Scientific Electronic Library Online (Scielo) SciELO e do site UFPR. Os resultados do estudo possibilitaram constatar divergências para atender os requisitos de navegação e interação dos usuários que acessaram e interagiram com os diferentes sites. $O$ estudo demonstrou que ambos os sites apresentaram falhas na usabilidade, contudo o site com maiores problemas de usabilidade foi o SciELO em comparação com o site mantido pela UFPR.
\end{abstract}

\section{Palavras-Chave}

Usabilidade. Website. Periódico científico.

\begin{abstract}
The present study presents a structured approach based on results obtained with the application of usability assessments of websites belonging to the Boletim de Ciências Geodésicas (BCG) journal, $B C G$ has two websites for communication with authors and readers, one is part of Scielo and another belongs to the Federal University of Paraná (UFPR), which maintains websites for its entire set of journals, including BCG. The premise established for carrying out the study was that different results are obtained with the application of usability assessments on both sites. The objective of the study was to evaluate the usability of the website of the Scientific Electronic Library Online (Scielo) SCIELO digital library and of the UFPR website. The results of the study made it possible to observe divergences to meet the navigation and interaction requirements of users who accessed and interacted with the different sites. The study showed that both sites had usability failures, however the site with the greatest usability problems was SCIELO compared to the site maintained by UFPR.
\end{abstract}

Key words 
Usability. Web site. Scientific journal.

\section{INTRODUÇÃO}

A Internet é um meio de comunicação e de entretenimento bastante utilizado no mundo, estando presente na vida profissional de um grande número de pessoas. Em atividades de ensino e pesquisa a Internet é fundamental, possibilitando aos pesquisadores acessarem, debaterem e compartilhar um vasto fluxo de informações. Contribuições importantes para a ciência são publicadas em periódicos científicos, que na atualidade estão hospedados em sites da internet (BRICKLEY et al., 2019). Estas publicações são acessadas e difundidas pela comunidade científica as quais possui por propósito de compartilhar o conhecimento, facilitando a construção e o aprimoramento de novas práticas apoiando-se em debates que estão em constante aperfeiçoamento (ORTINAU, 2011).

Em qualquer ambiente virtual, inclusive o pertencente aos periódicos científicos, a validação cognitiva desses ambientes é fortemente influenciada pelo design, acessibilidade e aceitabilidade por parte dos usuários. A usabilidade de um site está relacionada à rapidez e facilidade com que os usuários conseguem realizar suas tarefas (NIELSEN, 1993; ALCÁNTARA et al., 2019). Porém se a qualidade do site for considerada insatisfatória, devido a experiência do usuário não ser positiva, é muito provável que os usuários mudem para outra fonte de informação, trocando o site por outro (HAAKSMA et al., 2018).

A utilização de técnicas como o User Centred Design (UCD) e Experiência do Usuário (UX), na busca pela satisfação dos usuários por meio da elaboração de produtos utilizáveis e compreensíveis que atendam às suas necessidades e interesses (SALAH; PAIGE; CAIRNS, 2014). Com a finalidade de potencializar a aceitação e a produtividade dos sistemas interativos proporcionando um elevado grau de experiência por parte dos usuários, através de critérios de usabilidade avaliados com o emprego de técnicas específicas, atualmente existem critérios e padrões que orientam as avaliações de usabilidade, como a norma direcionada ao design centrado em usuários conhecida como ISO 9241-11 (2018).

Ao interagir com as interfaces, os usuários estabelecem um elo que permite a utilização das funcionalidades do sistema, ocorrendo uma familiarização com os processos que envolvem a interação dos usuários, minimizando a possibilidade do cometimento de equívocos (KOMARKOVA et al., 2019). É possível afirmar que a interface é a parte do sistema que possibilita ao usuário observar, tocar e sentir, portanto uma interface mal planejada pode ser um obstáculo para a aceitabilidade por parte dos usuários (ONEY et al., 2018). Segundo Kubícek et al., (2017), os problemas de usabilidade, muitas vezes podem ser resolvidos por exemplo com simplificações, para facilitar os processos cognitivos dos usuários, contudo para ser conhecido e solucionado problemas desta natureza, é necessário conhecê-los o que é possível com a realização de testes de usabilidade. A usabilidade de uma interface vai muito além de uma simples análise da interação entre o homem e a máquina, ela envolve inúmeros aspectos relacionados à cognição humana (MAIA et al., 2019).

Estudos como os de Miraz et al. (2017), ressaltam a importância da realização de avaliações de usabilidades em Web sites, sugerindo que aspectos importantes de usabilidade podem ser determinados, com base em metodologias que utilizam questionários para coletar dados. No estudo de Miraz et al. (2017), a análise por meio de questionário demonstrou desigualdades na satisfação dos usuários e uma insatisfação geral com a versão em outro idioma do mesmo site. Segundo Namoun et al. (2021), a usabilidade de sites Web é 
um fator crítico para a aceitação e satisfação dos visitantes, conforme o autor muitas vezes é praticamente ignorada falhas de usabilidade nos sites. Outro estudo que reforça a importância de avaliações de usabilidade foi a realização de avaliações em Web sites das Editoras Universitárias das regiões Sul, Centro-Oeste e Norte do Brasil com foco na eficácia, eficiência e satisfação, por De Lima et al. (2018), identificando problemas e propondo soluções visando otimizar a construção e disseminação do conhecimento na universidade.

Utilizar técnicas de avaliação de usabilidade permite compreender os pontos problemáticos de interação dos usuários com os Web sites (BECKER et al., 2018). O estudo de caso foi centrado no periódico Boletim de Ciências Geodésicas (BCG), que é um periódico trimestral destinado à publicação de artigos inéditos em inglês, na área das Ciências Geodésicas e áreas afins. O periódico BCG possui dois sites para comunicação com os autores e leitores, sendo que a revista faz parte da biblioteca digital Scientific Electronic Library Online (Scielo). Assim como a Universidade Federal do Paraná (UFPR) mantém um site para todo seu conjunto de revistas, incluindo o BCG. O artigo em foco explora características da produção científica acerca da usabilidade, com o problema pesquisado consistindo em verificar se a utilização dos diferentes sites do periódico BCG apresentam divergências para atender os requisitos de navegação e interação dos usuários. A premissa estabelecida foi que resultados distintos são obtidos com a aplicação de avaliações de usabilidade em ambos os sites. O objetivo do presente estudo foi avaliar a usabilidade do periódico BCG e comparar os resultados do site SciELO com o site pertencente à própria UFPR.

\section{AVALIAÇÃO DE USABILIDADE}

A usabilidade pode ser definida como a "capacidade de determinado sistema ser compreendido, operado e ser atraente aos usuários" (ISO 9241-11, 1998). Segundo Maia et al. (2019), a palavra "usabilidade", é um substantivo do adjetivo "utilizável". Em outras palavras, a usabilidade confere a facilidade de utilização e aprendizado dos usuários de um determinado sistema, por meio de métricas denominadas eficiências, eficácia e satisfação (BEVAN, 1995; DE LIMA et al., 2018). As avaliações de usabilidade podem ser realizadas empregando diferentes métodos (PUGLIESI et al., 2013). A seguir são apresentados métodos que são utilizados para coletar dados nos testes de usabilidade.

Entrevistas: são definidas como uma metodologia que envolve a interação verbal entre o pesquisador e os participantes com intenções específicas. Nielsen (1993) argumenta que entrevistas são adequadas para estudos exploratórios onde pouco se conhece acerca daquilo que se está procurando.

- Questionários: Os questionários são idealizados com propósito de serem divididos em uma série de indagações sobre um determinado tema, incluindo perguntas do tipo abertas ou fechadas. O questionário restringe as possibilidades de resposta e possíveis feedback dos usuários (KOVACIC e GREEN 2012).

- Observação dos usuários: Este método possibilita a descoberta de problemas de usabilidade, enquanto o usuário está interagindo com o sistema.

- Think Aloud: Os participantes executam ações de interação com a interface, expressando em voz alta seus pensamentos e sentimentos.

- Mensuração de desempenho: possui um grupo de usuários que executa um conjunto pré-definido de tarefas enquanto coletam informações sobre o tempo e os erros cometidos.

- Registro de dados: É uma técnica que envolve a existência de um sistema computa- 
cional que automaticamente colete estatísticas acerca do uso detalhado da interface a ser avaliada.

- Avaliação Heurística: Realizado por especialistas utilizando diretrizes com o propósito de identificar possíveis falhas no design.

Os testes podem ser moderados, correspondendo ao tipo de teste mais tradicional, ou não moderados. $O$ moderador é quem guia o usuário pelo roteiro do teste, realizando indagações e coletando dados (HERTZUM et al., 2015). Os testes de usabilidade nãomoderados correspondem a testes que podem ser realizados de forma totalmente remota através de ferramentas que auxiliem o usuário a executar as tarefas sem a presença do moderador. O Teste de Usabilidade Remoto Não Moderado (URUT), é uma técnica projetada para ajudar a superar as desvantagens do teste de usabilidade moderados, pois a observação moderada pode ser considerada intrusiva (SCHIRRA e ALLISON, 2018).

\section{TRATAMENTO E ANÁLISE DOS DADOS}

Os testes de usabilidade em geral são realizados por meio da aplicação de tarefas de uso simulando situações reais, porém a quantidade e qualidade dos dados a serem visualizados pode afetar o tempo de execução de um teste de forma significativa, com destaque para alguns fatores como processador, mouse, teclado, monitor, memória e formato da tela e uso de diferentes dispositivos, influenciam os resultados dos testes (PUGLIESI et al., 2013). Os dados provenientes dos métodos como questionários possibilitam verificar a taxa de acerto das interferências mediante a mudança de interface de visualização, permitindo inclusive análises estatísticas e a criação de gráficos para representar os resultados.

Uma análise dos dados quantitativa possibilita identificar possíveis dificuldades na utilização do produto, pode ser contabilizado o tempo que o usuário leva para realizar o teste, bem como número de clicks e rastreamento ocular. Na literatura existem diversos métodos utilizados para medir a usabilidade de forma quantitativa, como por exemplo a escala de resposta psicométrica com destaque para a escala de Likert (COOPER e HARPER, 1969; REID e NYGREN, 1988; COLEMAN, 1993; HARPER e NORMAN 1993; NIELSEN, 1993; SAURO e ZAROLIA, 2015).

Utilizando uma metodologia de interpretação e observação durante os testes, ou mesmo por meio da aplicação de questões discursivas nos questionários, é possível inferir impressões, sensações e preferências de uso dos participantes. Enriquecendo a avaliação de usabilidade com a coleta de dados qualitativos. Os dados qualitativos possibilitam verificar por exemplo a satisfação dos por meio de feedbacks, o que facilita nos processos de desenvolvimento e planejamento do sistema, que visam corrigir as falhas e melhorar os requisitos de uso e interação dos usuários com a interface.

\section{MATERIAL E MÉTODOS}

Esta etapa do trabalho contém uma descrição da coleta e organização dos dados, que possibilitaram realizar a avaliação de usabilidade dos diferentes Web sites pertencentes ao BCG. Existem variadas metodologias que são utilizadas para avaliações de usabilidade, neste estudo foi utilizado o método questionário remoto não moderados, possibilitando aos participantes participarem do estudo em seu próprio tempo e sem interferências do moderador. Em todos os testes, foram apresentados aos voluntários o Termo de Consentimento Livre e Esclarecido (TCLE), contendo explicações detalhadas sobre a execução dos 
testes e a liberdade de interromper sua participação sem qualquer ônus ao mesmo. A Figura 1 ilustra ambas as interfaces que foram testadas no estudo.

Figura 1 - Interfaces dos Web sites pertencentes ao BCG

SCIELO

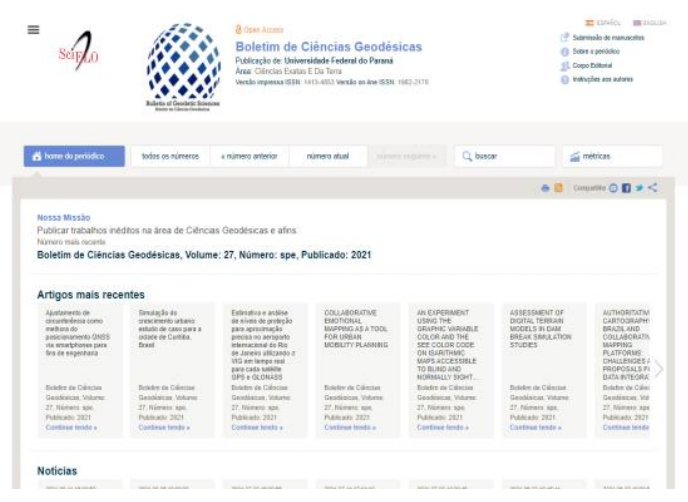

UFPR

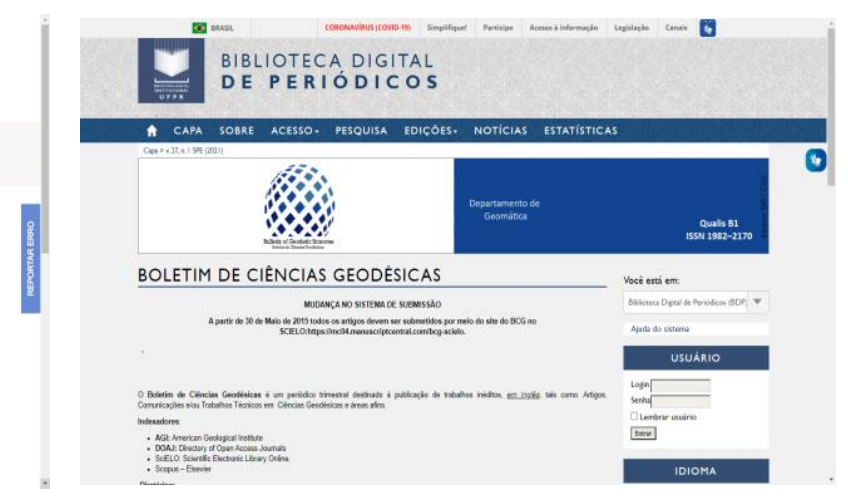

Fonte: Elaborado pelos autores (2021).

As técnicas utilizadas para recrutar os candidatos foram por meio de contato através de redes sociais e correio eletrônico, sendo que, no conteúdo das mensagens encaminhadas, foi realizado um breve esclarecimento sobre a pesquisa. Não foram feitas restrições quanto a gênero, raça ou credo, somente a faixa etária, que deveria ser acima de 18 anos e sem idade máxima. Foi encaminhado aos voluntários um link de acesso a uma lista de tarefas expostas na Tabela 1, disponibilizadas no Google Forms. O teste foi composto por 5 tarefas elaboradas e formatadas em estrutura de narrativas, as tarefas foram elaboradas com a finalidade de cobrir as principais funcionalidades de ambos os sites.

Tabela 1 - Tarefas elaboradas para aplicação do questionário

\begin{tabular}{c|l}
\hline \multicolumn{1}{c}{ Tarefas } \\
\hline 1 & $\begin{array}{l}\text { Entre no site do e realize uma busca pelo artigo de título "STRATEGY FOR } \\
\text { EXTRACTION OF FOURSQUARE'S SOCIAL MEDIA GEOGRAPHIC INFORMATION } \\
\text { THROUGH DATA MINING". Você conseguiu encontrar o artigo? } \\
\text { Encontre informações relacionadas ao Qualis do periódico. Foi possível en- } \\
\text { contrar esta informação? } \\
\text { Supondo que você tenha a necessidade de instruções para formatar seu } \\
\text { artigo respeitando as normas da revista, encontre essas informações no site. } \\
\text { Foi possível encontrar as instruções? } \\
\text { Com base nas informações contidas no site, você consegue concluir quais são } \\
\text { as linhas de pesquisa publicadas no BCG? } \\
\text { Supondo que você tomou a decisão de submeter o seu artigo no Boletim de } \\
\text { Ciências Geodésicas, encontre o template de formatação dos artigos. Foi } \\
\text { possível encontrar o template? }\end{array}$ \\
\hline
\end{tabular}

Fonte: Elaborado pelos autores (2021).

A elaboração do teste teve a finalidade de simular interações comuns no uso dos Web sites pertencentes ao BCG, apresentadas dentro do cenário específico, que simulasse a utilização "real" do site SciELO e do site UFPR. A realização das tarefas possibilitou a obtenção de resultados que permitiram a comparação entre os diferentes sites pertencentes ao periódico BCG. Quanto ao tamanho da amostra correspondente ao número de partici- 
pantes necessários para validar os resultados, o autor Nielsen (1993), afirma que a partir de cinco participantes para cada site, já é possível verificar parte significativa dos problemas de usabilidade, este número de participantes conforme Nielsen (1993), possibilita identificar cerca de $85 \%$ de falhas na interface avaliada.

\section{RESULTADOS E DISCUSSÃO}

Os resultados são apresentados sempre de forma compilada e generalizada. A caracterização dos voluntários considerou o grau de educação formal e área de formação dos participantes. O TCLE foi respondido por $100 \%$ dos participantes, aceitando participar da pesquisa de forma totalmente voluntária. Foram compilados e analisados os dados coletados de 13 participantes, no qual 6 voluntários avaliaram o site SciELO e 7 voluntários avaliaram o site da UFPR.

Os voluntários foram pessoas anônimas que aderiram ao estudo de forma totalmente livre, não foi realizado nenhuma objeção quanto a participação destas pessoas e sem controle do número de participantes, os dados coletados foram analisados separadamente e ao final foi realizado uma análise por meio de média, mediana e desvio padrão e não apenas valores absolutos, o que ajuda a minimizar e identificar possíveis "outliers" ou valores atípicos nas amostras.

Participaram do estudo de forma voluntária pessoas com nível de escolaridade categorizadas como graduação e mestrado, os voluntários que realizaram o teste utilizando o site SciELO, foram correspondentes a $83 \%$ mestres e $17 \%$ graduados. Relacionado aos participantes que avaliaram o site da UFPR, foi correspondente a $57 \%$ de mestres e $43 \%$ graduados.

Entre os participantes que realizaram a avaliação do site SciELO, 83\% possuíam formação na área de Engenheiro Cartógrafo e de Agrimensura, e 17\% em Ciências Geodésicas. Em relação a formação dos participantes que realizaram os testes avaliando o site da UFPR, 43\% possuíam a formação em Ciências Geodésicas, 14\% eram Engenheiros Cartógrafos e Agrimensores, $14 \%$ eram formados em Engenharia Agrícola e $29 \%$ possuíam formação em Ciências Agrárias.

Os voluntários que participaram do teste de usabilidade possuem formação na área que é realizado as publicações do BCG, portanto os participantes do estudo devem conhecer a revista, inclusive acessando e interagindo com o material produzido por ela. A Figura 2 contém gráficos correspondendo aos resultados da tarefa 1 presente na Tabela 1, a barra azul corresponde aos participantes que demonstraram sucesso na tarefa e a barra vermeIha corresponde ao insucesso. 
Figura 2 - Realizar busca por artigos

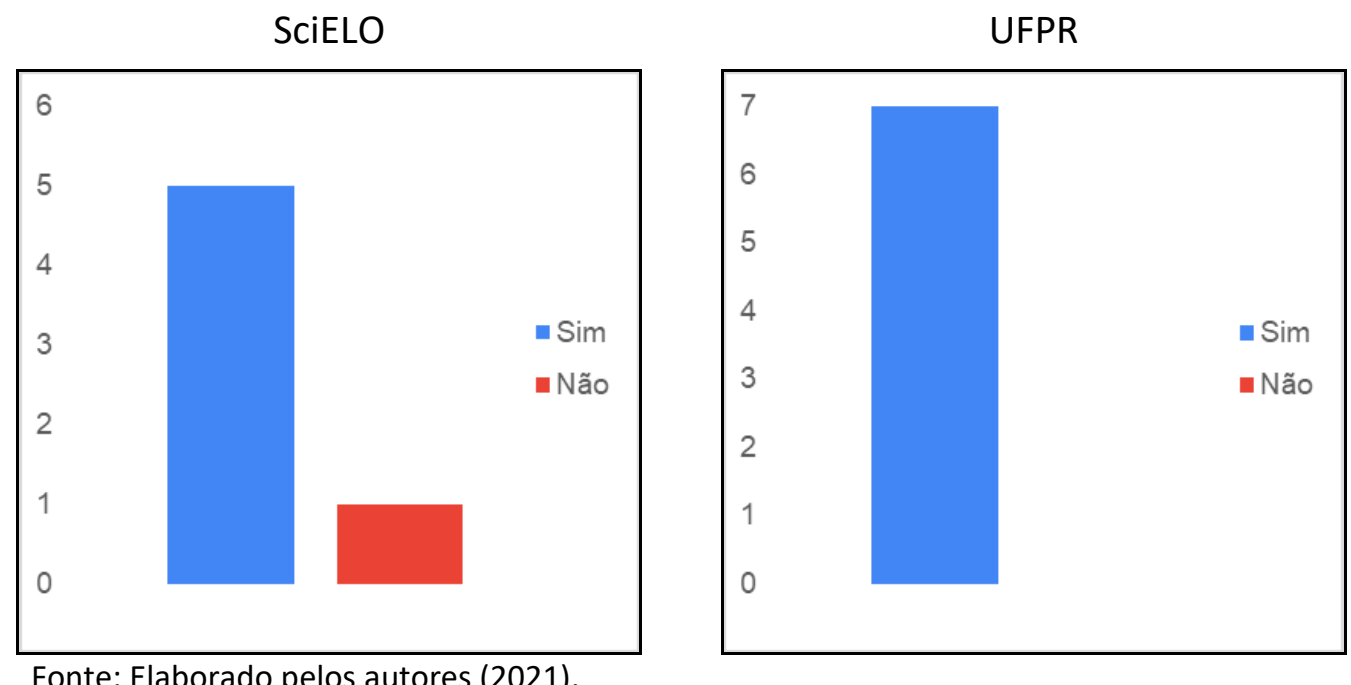

A Figura 2 contém respostas referentes à tarefa 1, que teve como propósito principal estimular os voluntários a utilizarem a ferramenta de buscas por artigos dentro de ambos os sites do BCG. Como resultados, foi quantificado que 5 participantes que avaliaram o site SciELO do BCG, conseguiram navegar e encontrar o respectivo artigo, por outro lado 1 participante não conseguiu encontrar o artigo. Em relação ao teste que foi aplicado no site UFPR do BCG, os resultados demonstraram que todos os 7 voluntários conseguiram encontrar o respectivo artigo. A Figura 3 contém resultados da tarefa 2, que teve por propósito estimular os participantes do teste a encontrar o Qualis do periódico BCG.

Figura 3 - Informações relacionadas ao Qualis do periódico BCG

SCIELO

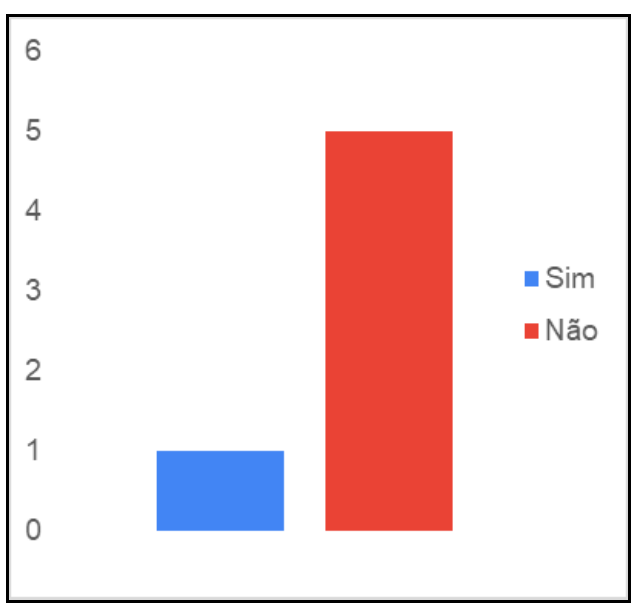

Fonte: Elaborado pelos autores (2021).
UFPR

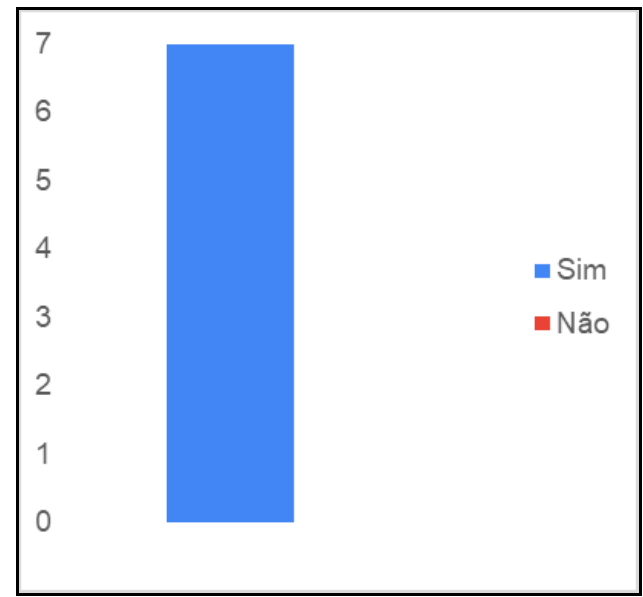

Conforme os resultados ilustrados na Figura 3, é possível constatar que 5 voluntários que avaliaram o site SciELO do periódico BCG, não conseguiram visualizar a informação referente ao Qualis da revista, com apenas 1 dos voluntários tendo sucesso nesta tarefa. Estes resultados demonstram que o site SciELO possui problemas significativos de visualização e disponibilidade de informações básicas referentes ao periódico BCG. Relacionado aos voluntários que realizaram a tarefa no site UFPR do BCG, todos os 7 participantes demonstraram êxito na tarefa. A Figura 4 contém resultados da aplicação da tarefa 3 da Ta- 
bela 1.

Figura 4 - Encontrar instruções para formatar o artigo

SCIELO

UFPR
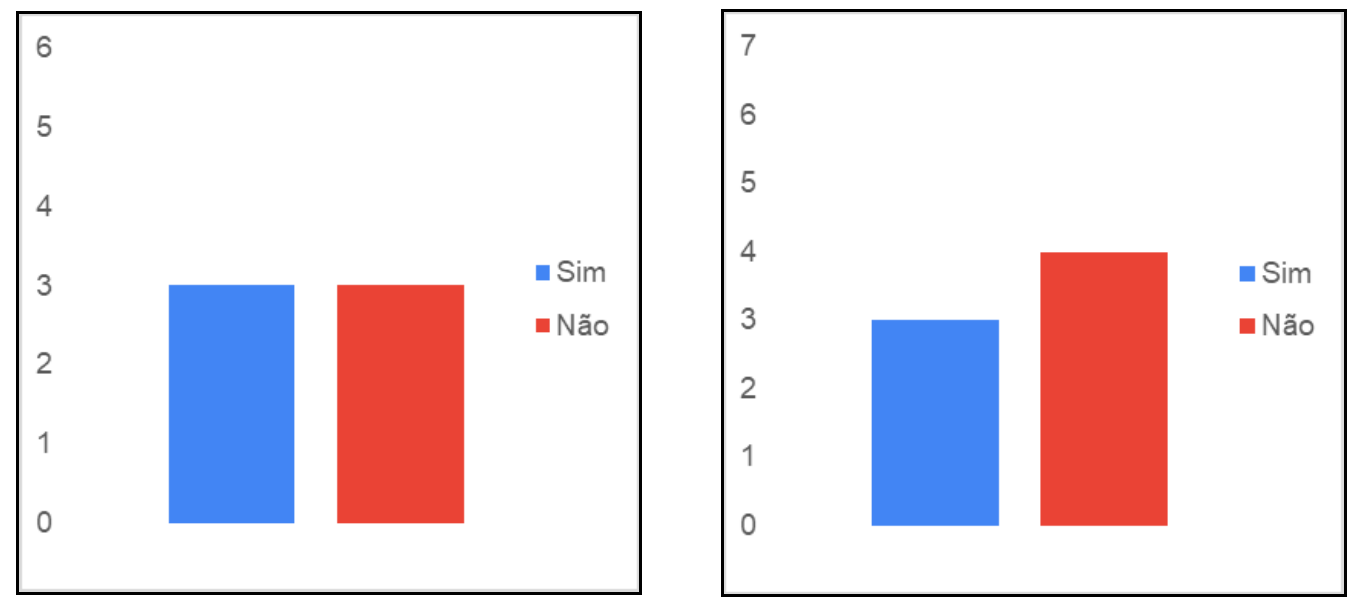

Fonte: Elaborado pelos autores (2021).

A tarefa 3 foi aplicada com o propósito de estimular os participantes a realizarem buscas por instruções para formatar um artigo, visando a adequação para com as normas do periódico BCG. Conforme os resultados apresentados nos gráficos da Figura 4, foi possível constatar que 3 dos voluntários conseguiram encontrar informações no site SciELO e 3 não conseguiram encontrar as respectivas informações. Relacionado ao site UFPR, foi constatado que 3 dos participantes obtiveram êxito na tarefa 3 e 4 pessoas não obtiveram sucesso. Os resultados em ambos os sites sugerem que parte significativa das pessoas que pretendem publicar um artigo no periódico BCG possivelmente estão se deparando com certas dificuldades para encontrar instruções que as ajudem a formatar o seus artigos. A Figura 5 apresenta os resultados da tarefa 4, Tabela 1.

Figura 5 - Linhas de pesquisa publicadas no BCG

\section{SCIELO}

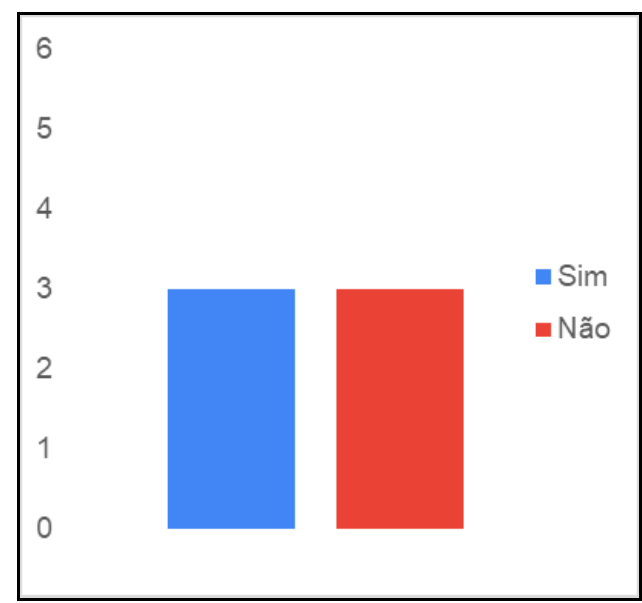

Fonte: Elaborado pelos autores (2021).
UFPR

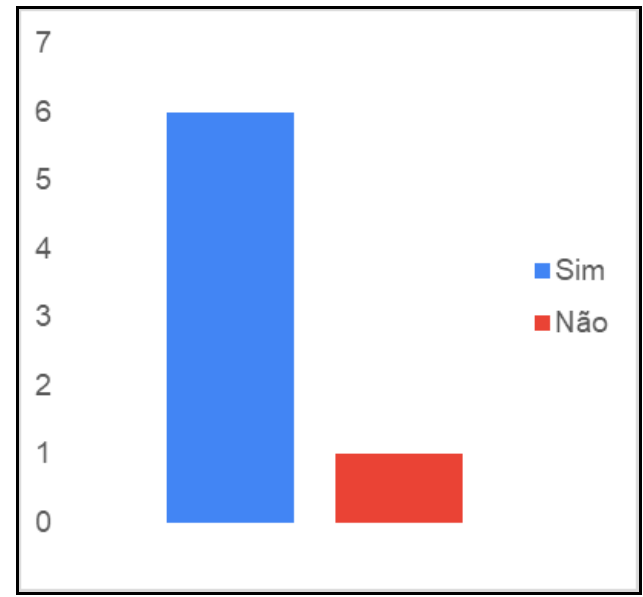

Conforme os resultados apresentados na Figura 5, é possível afirmar que 3 participantes que avaliaram o site SciELO conseguiram encontrar informações relacionadas a li- 
nha de pesquisa que são publicadas no periódico BCG e 3 não conseguiram obter estas informações. Com relação ao site UFPR pertencente ao BCG, foi constatado que 6 dos participantes encontraram as informações e apenas 1 dos voluntários não conseguiu extrair informações do site. Estes resultados reforçam as constatações anteriores de que o site SciELO possui problemas de acesso, visualização e possivelmente de disponibilidade de informações, em relação ao site UFPR que demonstrou resultados positivos. A Figura 6 contém os resultados correspondentes a tarefa 5, Tabela 1.

Figura 6 - Localizar o template para formatar artigos
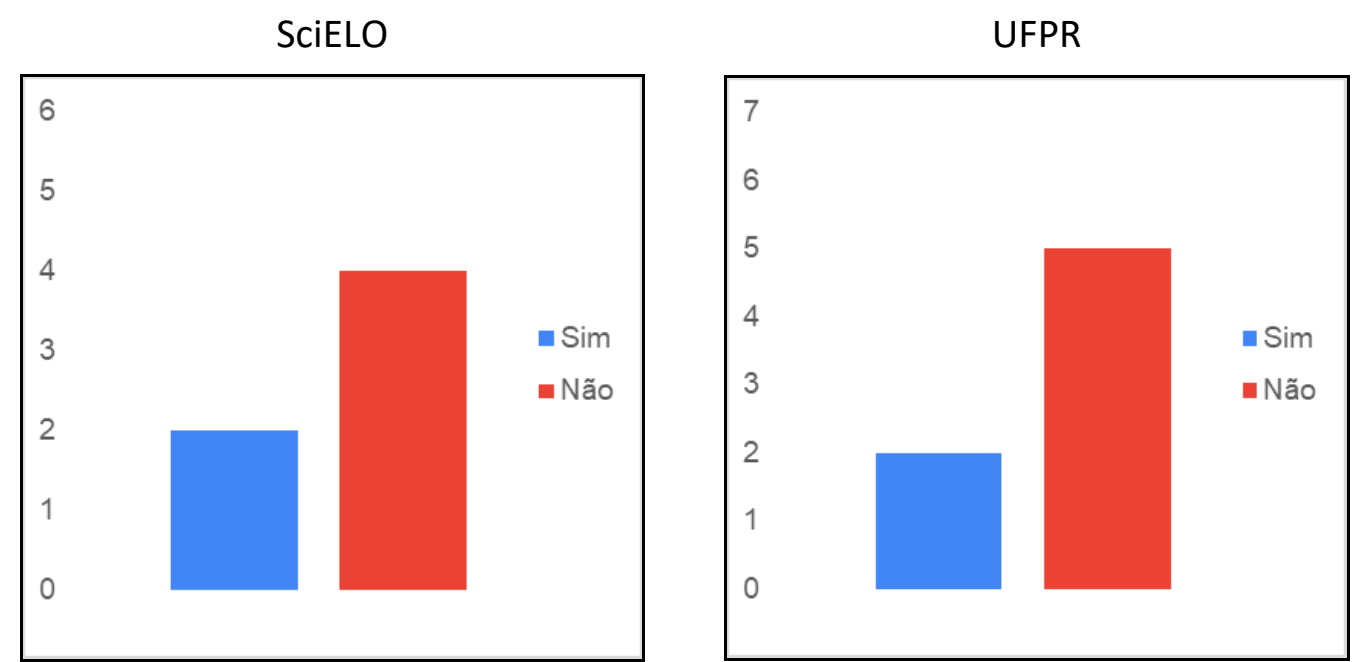

Fonte: Elaborado pelos autores (2021).

Com base nos resultados presentes na Figura 6, foi possível verificar que cerca de 4 das pessoas voluntárias que utilizaram o site SciELO não localizaram o template, e 2 voluntários conseguiram localizar o template. Em relação aos participantes que utilizaram o site UFPR, foi verificado que 5 pessoas não conseguiram encontrar o template e 2 pessoas conseguiram localizá-lo. Estes resultados sugerem problemas significativos em ambos os sites de fornecer acesso ao template do periódico BCG, este fato pode fazer com que as pessoas que queiram publicar seus manuscritos no BCG encontre dificuldades significativas para adequar seus artigos considerando as normas da revista e atendendo de forma satisfatória as regras de formatação para submissão dos artigos.

Os dados obtidos com a aplicação das tarefas foram submetidos a uma análise estatística descritiva, que possibilitou uma comparação geral entre a usabilidade de ambos os sites acessados. A Figura 7, contém dados como mínimo, máximo, primeiro quartil, segundo quartil ou mediana e o terceiro quartil, conforme os resultados estatísticos foi possível constatar que a performance dos participantes que acessaram o BCG pelo site SciELO apresentaram desempenho inferior em comparação com o acesso ao BCG e utilizando o web site UFPR. 
Figura 7 - Comparação entre os desempenhos nas tarefas realizadas nos Web sites

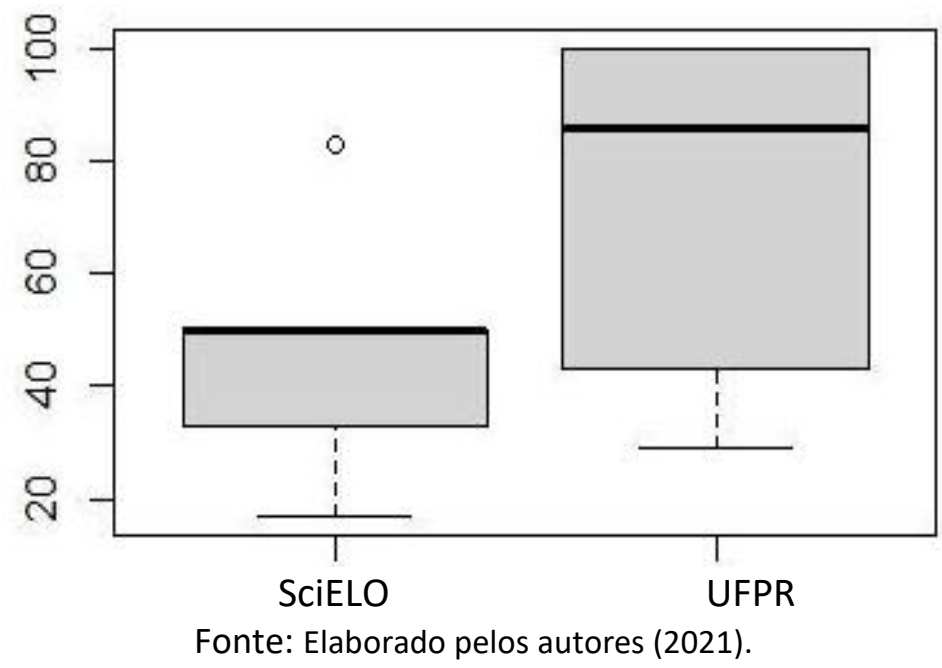

Os dados não foram submetidos a análises estatísticas conclusivas por meio da utilização de testes hipóteses, em decorrência do tamanho amostral dos voluntários que aderiram ao estudo não ser suficiente. Após a realização das tarefas de interação foi solicitado aos participantes informar o tempo médio estimado gasto para realização das tarefas, a Tabela 2 contém os resultados.

Tabela 2 - Medidas estatísticas do tempo gasto para realizar das tarefas

\begin{tabular}{lll}
\hline Medidas estatísticas & SciELO & UFPR \\
\hline Média & $9 \mathrm{~min}$ & $8 \mathrm{~min}$ \\
Desvio padrão & $5,29 \mathrm{~min}$ & $4,07 \mathrm{~min}$ \\
\hline
\end{tabular}

Fonte: Elaborado pelos autores (2021).

Como resultado comparativo entre ambos os sites foi possível inferir que o SciELO demandou mais tempo médio de acesso para realização das tarefas, em relação ao site da UFPR. O desvio padrão que indica a distância média entre a variável e a média aritmética da amostra foi maior no site SciELO, em comparação com as mesmas tarefas realizadas no site da UFPR. Após a realização das tarefas, foi solicitado aos participantes que descrevessem sua opinião de forma discursiva a respeito das impressões, sensações, pontos positivos e negativos, a respeito da experiência que foi obtida ao acessar os Websites pertencentes ao BCG. As respostas obtidas foram computadas na no Quadro 1.

Quadro 1 - Opinião dos participantes que avaliaram os sites do BCG

\begin{tabular}{l} 
Web site: SciELO \\
\hline O site contém poucas informações de orientação à submissão (template, tutorial \\
de submissão, orientação para elaborar cover letter, rebuttal letter etc). Não consegui \\
encontrar informações sobre a revista acessando apenas o site da Scielo, para obter tais \\
informações foi necessário entrar no site da UFPR. No geral, como ponto positivo, achei \\
o banco de dados para consultar artigos muito bom e de fácil acesso. Porém, como pon- \\
to negativo, creio que a barra de navegação do site poderia ser mais intuitiva e no home \\
do site poderia ter as informações do Qualis da revista. \\
\hline O site pesquisado parece estar em processo de construção. A interface é estetica- \\
mente agradável.
\end{tabular}




\section{Site confuso, páginas sem informações}

A maioria das informações foram encontradas em uma única aba do site, porém as informações como o Qualis por exemplo deveriam estar em maior destaque.

Diante do teste realizado muitas poucas informações foram de fácil acesso. Ao logar no site da Scielo já se encontra a versão em português, na qual a maioria das informações aparece a seguinte informação: "Informação não disponível. Por favor, consulte a versão do periódico em inglês." Sugiro no final desta frase colocar o link ou automaticamente redirecionar a página.

O template, as linhas de pesquisa publicadas no BCG e o Qualis do periódico poderiam ser apresentados de forma mais clara e objetiva no site.

A princípio achei o site com pouquíssimas informações de orientação à submissão.

O site pesquisado parece estar em processo de construção. A interface é esteticamente agradável.

Site confuso, páginas sem informações

\section{Web site:UFPR}

Dificuldade de encontrar o tamplete

O site parece estar desatualizado e as informações com as instruções estão na plataforma da SCIELO.

Muito boa organização a interface do site

$\mathrm{O}$ site me pareceu confuso e pouco intuitivo. O teste abordou aspectos do contexto de uso de um leitor e de um autor de artigos científicos, explorando necessidades básicas, onde o site se mostrou com baixa eficiência nestas resoluções.

O site é bastante intuitivo, de fácil interpretação, porém ocorreu um erro na primeira tentativa de abrir o link de diretrizes para autores, o que foi corrigido na segunda tentativa, redirecionando para outra página do site.

O Qualis do periódico está bem visível, de modo que esta é importante para os autores interessados em publicar.

Existe uma nota sobre publicações após 2015, não acho que seja mais necessário.

Fonte: Elaborado pelos autores (2021).

Conforme a opinião discursiva dos participantes foi possível constatar significativa insatisfação dos participantes que utilizaram o site da SciELO, com opiniões que apontam para a falta de informações de orientação à submissão e falta de informações sobre o periódico BCG, com destaque para dificuldades para encontrar informações sobre o Qualis da revista e encontrar o template. No site da UFPR foi verificado feedbacks positivos, como ressaltando que o Qualis do periódico está bem visível, ou mesmo que o site é intuitivo de ser utilizado e elogios referentes a organização da interface do site. As opiniões corroboram com os resultados obtidos com a aplicação das tarefas, onde em sua maioria os participantes demonstraram melhor desempenho ao utilizar o site da UFPR em comparação com os participantes que utilizaram o site SciELO.

\section{CONSIDERAÇÕES FINAIS}

Com a realização dos testes de usabilidade em ambos os sites que possibilitam acesso ao periódico BCG, foi possível constatar divergências para atender os requisitos de navegação e interação dos usuários. Por meio da realização do presente estudo foi possível comprovar que resultados distintos foram obtidos com a aplicação das avaliações de usabilidade nos sites SciELO e UFPR. A partir da coleta e análise dos resultados é possível conclu- 
ir que o site com maiores problemas de usabilidade foi o SciELO em comparação com o Web site mantido pela UFPR, que apresentou melhor desempenho na maioria das tarefas.

Porém ambos os sites demonstraram problemas, com falhas na usabilidade, como por exemplo falta de informações e dificuldade para encontrar informações desejadas, problemas para localizar o template do periódico e dificuldade para visualizar as linhas de pesquisa aceitas para publicação e o Qualis da revista.

Outro ponto negativo foi a existência simultânea de ambos os sites para os mesmos propósitos, que acabam confundido os pesquisadores leitores que desejam submeter um artigo ou apenas realizar a leitura, fazendo com que o usuário tenha dificuldade para assimilar qual site deve acessar, bem como a atualização e melhorias dos sites não são sincronizadas, produzindo divergências estéticas e de usabilidade entre eles.

\section{AGRADECIMENTOS}

O primeiro autor agradece a Coordenação de Aperfeiçoamento de Pessoal de Nível Superior (CAPES) pelo incentivo a pesquisa e apoio financeiro, Processo $\mathrm{n}$ 은 88887.601430/2021-00.

\section{REFERÊNCIAS}

ALCÁNTARA, P. J. M.; BLANCO, E. F. J.; ARMENSKI, T.; DEL BARRIO, G. S. The antecedent role of online satisfaction, perceived risk online, and perceived website usability on the affect towards travel destinations. Journal of Destination Marketing \& Management, v. 9, p. 2035, 2018. doi.org/10.1016/j.jdmm.2017.09.005.

BECKER, V. G. D.; RAMOS, T. S.; BEZERRA, E. P. Design Audiovisual: a interseção dos estudos de audiência com a Interação Humano-Computador. Conexão-Comunicação e Cultura, v.17, 2018. DOI 10.18226/21782687.v17.n33.13.

BEVAN, N. Human Computer Interaction Standards. Elsevier, V.20, p.885-890, 1995. DOI doi.org/10.1016/S0921-2647(06)80326-6.

BRICKLEY, D.; BURGESS, M.; NOY, N. Google Dataset Search: Building a search engine for datasets in an open Web ecosystem. IN: THE WORLD WIDE WEB CONFERENCE, p.1365-1375, 2019. DOI doi.org/10.1145/3308558.3313685.

COLEMAN, N. S. (Software Usability Measurement Inventory) as a knowledge elicitation tool for improving usability. Unpublished BA Honours thesis, Dept. Applied Psychology, University College Cork, Ireland, 1993.

COOPER, G.E., HARPER, R. P. The use of pilot ratings in the evaluation of aircraft handling qualities (NASA Ames Technical Report NASA TN - D - 5153). Moffett Field, CA: NASA Ames Research Center, 1969.

DE LIMA, I. F.; DE LIMA, R. F.; SILVA, M. C.; MEDEIROS, V. H. G. Avaliando a usabilidade dos 
websites de editoras universitárias brasileiras. Ciência da Informação em Revista, v.5, p.4253, 2018. DOI: https://doi.org/10.22478/ufpb.1981-0695.2019v14n1.44361.

HAAKSMA, T. R..; DE JONG, M. D. T; KARREMAN, J. Users personal conceptions of usability and user experience of electronic and software products. IEEE TRANSACTIONS ON PROFESSIONAL COMMUNICATION, v. 61, p.116-132, 2018. DOI: 10.1109/TPC.2018.2795398.

HARPER, B. D., NORMAN, K. L. Improving user satisfaction: The questionnaire for user interaction satisfaction version 5.5. IN: PROCEEDINGS OF THE 1ST ANNUAL MID-ATLANTIC HUMAN FACTORS CONFERENCE. p.224-228, 1993.

HERTZUM, M.; BORLUND, P.; KRISTOFFERSEN, K. B. What do thinking-aloud participants say? A comparison of moderated and unmoderated usability sessions. International journal of human-computer interaction, v.31, p.557-570, $2015 . \quad$ DOI doi.org/10.1080/10447318.2015.1065691.

ISO 9241-11. Ergonomics of human-system interaction. Part 11: Usability: Definitions and concepts. 2018.

KOMARKOVA, J.; SEDLAK, P.; STRUSKA, S.; DYMAKOVA, A. Usability Evaluation the Prague Geoportal: Comparison of Methods. In 2019 INTERNATIONAL CONFERENCE ON INFORMATION AND DIGITAL TECHNOLOGIES (IDT), p.223-228, 2019. DOI: 10.1109/DT.2019.8813723.

KOVACIC, Z. J.; GREEN, J. S. Automatic Grading of Spreadsheet and Database Skills. Journal of Information Technology Education: Innovations in Practice, v.11, p.53-70, 2012. $10.28945 / 1562$.

KUBÍCEK, P.; SASINKA, C.; STACHON, Z.; STERBA, Z.; APELTAUER, J.; URBÁNEK, T. Cartographic design and usability of visual variables for linear features. The Cartographic Journal, v.54, p.91-102, 2017. DOI https://doi.org/10.1080/00087041.2016.1168141.

MAIA, M. A. Q.; BARBOSA, R. R.; WILLIAMS, P. Usabilidade e experiência do usuário de sistemas de informação: em busca de limites e relações. Ciência da Informação em Revista, v. 6, n. 3, p. 34-48, 2019. DOI doi.org/10.28998/cirev.2019v6n3c.

MIRAZ, M. H.; ALI, M.; EXCELL, P. Multilingual website usability analysis based on an International user survey. Human-Computer Interaction, p.236-244, 2017. DOI 10.13140/2.1.1208.8648.

NAMOUN, A.; ALREHAILI, A.; TUFAIL, A. A Review of Automated Website Usability Evaluation Tools: Research Issues and Challenges. IN: INTERNATIONAL CONFERENCE ON HUMANCOMPUTER INTERACTION. Springer, Cham, p.292-311, 2021. DOI doi.org/10.1007/978-3030-78221-4_20.

NIELSEN, J. Usability Engineering. 1993. 
ONEY, S.; LUNDGARD, A.; KROSNICK, R.; NEBELING, M.; LASECKI, W. S. Arboretum and arbility: Improving web accessibility through a shared browsing architecture. IN THE 31ST ANNUAL ACM SYMPOSIUM ON USER INTERFACE SOFTWARE AND TECHNOLOGY, p.937-949, 2018. DOI https://doi.org/10.1145/3242587.3242649.

ORTINAU, D. J. Writing and publishing important scientific articles: A reviewer's perspective. Journal of Business Research, v. 64, n. 2, p. 150-156, 2011. DOI https://doi.org/10.1016/j.jbusres.2010.02.002.

PUGLIESI, E. A., DECANINI, M. M. S., RAMOS, A. P. M., TSUCHIYA, Í.. Métodos para Avaliação da Usabilidade de Sistemas de Navegação e Guia de Rota. Revista Brasileira de Cartografia, v.65, 2013.

REID, G.B., NYGREN, T.E. The subjective workload assessment technique: A scaling procedure for measuring mental workload. In P.A. Hancock \& N. Meshkati. Elsevier, p.185-218, 1988. https://doi.org/10.1016/S0166-4115(08)62387-0.

SALAH, D.; PAIGE, RICHARD F.; CAIRNS, P. A systematic literature review for agile development processes and user centred design integration. PROCEEDINGS OF THE 18TH INTERNATIONAL CONFERENCE ON EVALUATION AND ASSESSMENT IN SOFTWARE ENGINEERING EASE '14, [s.l.], p.1-10, 2014. DOI https://doi.org/10.1145/2601248.2601276.

SAURO, J., ZAROLIA, P. A comprehensive measure of the quality of the website user experience. Journa'I of Usability Studies, v.10, p.68-86, 2015.

SCHIRRA, S., ALLISON, C.. I know what the companies are wanting more of: Professional Participants in Online Usability Studies. IN EXTENDED ABSTRACTS OF THE 2018 CHI CONFERENCE ON HUMAN FACTORS IN COMPUTING SYSTEMS. Montreal, Canada. 2018. DOI https://doi.org/10.1145/3170427.3188681 